\title{
The Effect of Seasonality on the Application of Accumulated Degree-Days to Estimate the Early Post-Mortem Interval
}

\author{
Stephanie B. Giles, Karl Harrison, David Errickson \& Nicholas Márquez-Grant
}

\begin{abstract}
This study investigated the effect of seasonal variables on decomposition in the early post-mortem period using 26 donated human cadavers at the University of Tennessee's Anthropology Research Facility (ARF), USA. The rate and pattern of decomposition in human cadavers (as measured by TBS and the revised TBSsurf methods) did not vary significantly between all seasons. Summer and autumn cadavers had comparable rates of accelerated decomposition despite significant differences in both ADD and temperature $(p<0.05)$. Spring cadavers had the slowest onset of decomposition characteristics, even compared to the few decomposition characteristics expressed in winter. Seasonal variation in humidity, rather than temperature, may be the overarching driving force for decomposition progression in the early post-mortem period. Both TBS and TBSsurf methods were poor predictors of the PMI $\left(R^{2}=0.4\right)$ and significantly over-estimated the PMI across all seasons, although to a lesser extent in spring. This study also demonstrated no relationship between known ADD and TBS/TBSsurf $\left(R^{2}=0.025\right)$. TBS and TBSsurf are ADD-based PMI estimation models that cannot be validated under experimental conditions. Accounting for seasonal expression of individual decomposition characteristics is needed for improvement of PMI predictability in forensic practice.
\end{abstract}

\section{Key Words}

Decomposition; ADD; PMI; TBS; Forensic Taphonomy 


\section{Introduction}

The ability to accurately estimate the post-mortem interval (PMI) from the observable state of human decomposition is a long-standing goal in the experimental work of forensic taphonomists, and in the casework of forensic pathologists and forensic anthropologists. An accurate PMI can provide information of critical significance in police investigations [1,2]. In the United Kingdom, the first 72 hours of a homicide investigation is considered a critical period that must maximise opportunities to secure ubiquitous evidence that is admissible in court [3]. This time period correlates to the 'early post-mortem' interval of the deceased where early post-mortem biochemical markers (PBMs) from the body have been extensively studied to determine the PMI and consequently assist in suspect and/or victim identification $[4,5]$.

Despite the plethora of research into the development of experimental techniques of PBMs a recent review found that all PBMs, including the highly researched vitreous humor potassium concentration, are not standardised in the forensic pathologist's estimation of the PMI in casework and do not currently qualify as evidence in court [6]. The assessment of early post-mortem physiological changes such as livor mortis and rigor mortis, also confer little predictive power due to their high variability of onset between individuals [7]. The initial absence of insect larvae, which may render entomological aging methods inapplicable until 72 hours post-death [8], also affirms the need to develop minimum PMl estimation models that are applicable to the early post-mortem period. Such models could corroborate circumstantial police evidence such as CCTV and mobile phone activity, to predict narrow PMI intervals with a high degree of accuracy.

Beyond the early post-mortem period, where remains may have been concealed or a lack of social contact precludes the discovery of the deceased, uncertainty increases regarding the PMI estimation due to the complexities of the human decay process. Numerous early researchers sought to classify soft tissue decomposition into stages that correlate with the PMI [9-11]. Galloway et al [12] landmark decay sequence of 'Fresh', 'Early', 'Advanced', 'Skeletonized' and 'Extreme' decomposition stages, was developed by observing surface depositions in an arid environment. This sequence, which has laid the foundations of revised decomposition scoring models in forensic taphonomy [13-16], confers limited applicability when estimating the PMI in specific geographical regions, reiterating that decomposition is an environmentallydependant process.

While there is a surfeit of environmental and intrinsic variables known to affect the decomposition process, temperature is perhaps the most extensively studied seasonal variable over recent decades. This is not surprising when we consider that temperature has been canonised in the forensic taphonomy literature as 
the most influential 'independent' variable affecting the rate of soft tissue decay [2,17]. However, quantifying the effects of temperature on human decomposition is largely complexed by its inter-relationship with other taphonomic seasonal variables such as air humidity, rainfall and sun exposure [18-20]. Given the considerable differences in temperature, rainfall and humidity across the four seasons in temperate locations (spring, summer, autumn and winter) and the two seasons in tropical areas (monsoon and dry), the effect of seasonal variation on decomposition is relatively under-explored in the taphonomic literature [20-21].

The use of accumulated degree days (ADD), which is routinely used in the practice of forensic entomology to predict insect development [8], has provided a solution to model the effects of temperature on decomposition. Predictive formulas have been devised to provide estimated ADD values based on observed levels of accumulated decomposition, which are then used to back-calculate a PMI $[13,15,16]$. Megyesi et al [13] demonstrated that estimated ADD could account for approximately $80 \%$ of the variability in the human decay rate, concluding that temperature is a more reliable predictor of the PMI than the accumulated level of decomposition, referred to as the 'Total Body Score' (TBS) [13]. Studies have reported vastly conflicting findings regarding the contribution of $A D D$ to the progression of the decay rate, with quantification of this effect between 25-94\% [22-25]. These inconsistencies, coupled with discrepancies between the ambient temperature at the site of body deposition and retrospective temperature recordings from local weather stations [22], affirm the variability inherent in the quantification of temperature effects on decomposition.

Numerous validation studies of the Megyesi et al [13] equation report equally low success rates in estimating the PMI under experimental conditions, finding significant overestimations of the calculated ADD compared to the actual ADD $[21,26]$. This is likely attributable to the assumption that the dependent variable (level of decay) can be used to predict the independent variable (ADD), a mistake correctly identified by Moffat et al [16]. Moreover, when the Megyesi et al [13] formula was applied in practice to non-government casework a broad PMI range between 22-122 days was reported [27]. This arguably confers a range too wide for any valuable forensic homicide application, where narrower and more accurate PMI intervals are required. Collectively, these errors currently confound the use of ADD models to estimate the PMI in forensic investigation practice.

Alternative research has suggested that quantifying the level of decomposition over time is a more valuable predictor of the PMI than ADD [15,25]; whilst other studies elucidate that neither ADD nor PMI are significantly influential variables of decomposition $[19,28]$. It is therefore recognised that a revision of the use of ADD is required when predicting the PMI from decaying human cadavers for it to be of any potential value in forensic casework. One such study by Moffatt et al [16] has addressed the limitations of the Megyesi et al [13] statistical model and revised the original formula by removing outlying data to produce more accurate prediction intervals of the PMI. There are few studies that have tested this revised model under experimental conditions. One study found it was more accurate at estimating of the PMI in the early decomposition stage [21]. However, this relied on porcine samples, which are arguably not a suitable proxy for human decomposition [29,30], and as yet, no study (to the authors' knowledge) has tested the Moffat et al [16] revised formula using human cadavers.

This study utilised donated human cadavers to compare the accuracy of two methods that incorporate ADD in their estimation of the early PMI: i) the Megyesi et al [13] TBS method and ii) the Moffatt et al [16] revised 
TBSsurf. In this study, both scoring methods were compared using human cadavers across the four temperate seasons (spring, summer, autumn and winter) to assess the seasonal variation in the decomposition rate as previously recommended [21]. The application of these ADD-based PMI estimation models to the early post-mortem period is of critical importance. This time frame correlates to the initial stages of forensic homicide investigations where an accurate PMI may contribute to suspect identification [3]. Forensic entomology methods are not routinely considered usable until 3 days post-death [8] and the current lack of standardised early PBM's in forensic pathology [6] provides further scope for this assessment.

\section{Material and Methods}

This study utilized a sample of 26 human cadavers of known PMI from the University of Tennessee's Body Donation Program. The experimentation site was the Anthropology Research Facility (ARF) in Knoxville, Tennessee, USA. The ARF is approximately 3 acres of densely populated deciduous woodland enclosed by razor-wire fencing and is located on the south bank of the Tennessee River. Prior to placement, the cadavers were stored in a $3^{\circ} \mathrm{C}$ morgue cooler for no less than 24 hours, to equilibrize internal body temperature. The cadavers were then placed 'fresh' on clean ground within the enclosure between January and October over a two-year period (2013 - 2015). For the purpose of this paper, "1 post-placement day" will be referred to as "1 PMI day", and so forth. Only the first 7 PMI days were studied to enable the expression of early to moderate decomposition changes that are frequently encountered in the early post-mortem period. The termination of 'moderate' decomposition (marked by the rupture of abdominal 'bloating' gases and darkening of the skin) generally occurs around 7 days in dry hot environments akin to Tennessee climatology of hot, humid summers, and mild winters [12]. All cadavers were approximately $\geq 20 \mathrm{~m}$ apart from each other and had little exposure to direct sunlight under the dense canopy cover of maple, oak and hickory trees.

The sample consisted of adult males $(n=15)$ and adult females $(n=11)$ with an age range of 42 - 80 years. All cases had natural causes of death with no injuries present. All cadavers were surface depositions that had been placed unclothed on the ground of the open wooded enclosure of the facility in a supine $(n=19)$ or prone position ( $n=7)$ (Table 1). Of these cadavers, some had been protected under wire cages to prevent scavenging by racoons $(n=6)$ [30], whislt others were covered with plastic tarp $(n=7)$, which is commonly used to accelerate the decomposition of mummified remains by facilitating entomology activity [30]. The remaining cadavers formed the 'control' group and were left uncovered $(n=13)$. To assess the effect of seasonality on the accumulation of decomposition, the cadavers were placed at various times of the year spanning all seasons: spring $(n=5)$, summer $(n=10)$, autumn $(n=7)$ and winter $(n=4)$. There was at least one 'control' (uncovered) cadaver in each seasonal trial. This allowed for comparisons of coverings within, and between, seasons. Where possible, cadavers were also placed on the same date within each season: spring $(n=4)$, summer $(n=8)$, autumn $(n=6)$ and winter $(n=2)$ to facilitate direct comparisons of taphonomic observations between cadavers. All months of the calendar year were represented in the dataset excluding February, March, November and December (Table 1).

\begin{tabular}{|c|c|c|c|c|c|c|}
\hline Case & Sex & Position & Covering & $\begin{array}{c}\text { Date of } \\
\text { Placement }\end{array}$ & $\begin{array}{l}\text { Season of } \\
\text { Placement }\end{array}$ & $\begin{array}{c}\text { Method of } \\
\text { Assessment }\end{array}$ \\
\hline
\end{tabular}




\begin{tabular}{|c|c|c|c|c|c|c|}
\hline 1 & $\mathrm{M}$ & Supine & Covered & January $15^{\text {th }}$ & Winter & Photographs \\
\hline 2 & $M$ & Supine & Covered & January 22nd & Winter & Photographs \\
\hline 3 & $\mathrm{~F}$ & Supine & Covered & January 23rd & Winter & Photographs \\
\hline 4 & $\mathrm{~F}$ & Supine & Uncovered & January $23^{\text {rd }}$ & Winter & Photographs \\
\hline 5 & $\mathrm{M}$ & Supine & Covered & April $15^{\text {th }}$ & Spring & Photographs \\
\hline 6 & $\mathrm{M}$ & Supine & Uncovered & May 1st & Spring & Photographs \\
\hline 7 & $\mathrm{~F}$ & Supine & Uncovered & May 1st & Spring & Photographs \\
\hline 8 & $\mathrm{M}$ & Prone & Uncovered & May $17^{\text {th }}$ & Spring & Photographs \\
\hline 9 & $\mathrm{M}$ & Prone & Uncovered & May $17^{\text {th }}$ & Spring & Photographs \\
\hline 10 & $\mathrm{M}$ & Supine & Uncovered & June $6^{\text {th }}$ & Summer & Visual \\
\hline 11 & $\mathrm{~F}$ & Supine & Uncovered & June $6^{\text {th }}$ & Summer & Visual \\
\hline 12 & $\mathrm{~F}$ & Supine & Uncovered & June $12^{\text {th }}$ & Summer & Visual \\
\hline 13 & $\mathrm{~F}$ & Supine & Uncovered & June $12^{\text {th }}$ & Summer & Visual \\
\hline 14 & $\mathrm{M}$ & Supine & Uncovered & July $15^{\text {th }}$ & Summer & Photographs \\
\hline 15 & $\mathrm{M}$ & Supine & Cage & July $21^{\text {st }}$ & Summer & Photographs \\
\hline 16 & $\mathrm{M}$ & Prone & Covered & August $11^{\text {th }}$ & Summer & Photographs \\
\hline 17 & $\mathrm{M}$ & Prone & Covered & August $11^{\text {th }}$ & Summer & Photographs \\
\hline 18 & $\mathrm{~F}$ & Prone & Uncovered & August $11^{\text {th }}$ & Summer & Photographs \\
\hline 19 & $\mathrm{M}$ & Prone & Uncovered & August $11^{\text {th }}$ & Summer & Photographs \\
\hline 20 & $\mathrm{~F}$ & Supine & Cage & September $23^{\text {rd }}$ & Autumn & Both \\
\hline 21 & $\mathrm{M}$ & Prone & Uncovered & September 23rd & Autumn & Both \\
\hline 22 & $\mathrm{M}$ & Supine & Covered & September $28^{\text {th }}$ & Autumn & Photographs \\
\hline 23 & $\mathrm{M}$ & Supine & Cage & October $15^{\text {th }}$ & Autumn & Visual \\
\hline 24 & $\mathrm{~F}$ & Supine & Cage & October $15^{\text {th }}$ & Autumn & Visual \\
\hline 25 & $\mathrm{~F}$ & Supine & Cage & October $15^{\text {th }}$ & Autumn & Visual \\
\hline 26 & $\mathrm{~F}$ & Supine & Cage & October $15^{\text {th }}$ & Autumn & Visual \\
\hline
\end{tabular}

Table 1 Sample Composition of 26 human cadavers observed over the first 7 days postplacement

The decomposition state was recorded from full-length photographs and/or by visual observations of the deceased using: i) the Megyesi et al [13] method of TBS and ADD, and ii) the Moffat et al [16] revised TBSsurf and ADD equation. Visual observations were undertaken daily for 10 cadavers throughout the study duration (Table 1). For cases where visual examination was not possible, case photographs were accessed from the ARF's daily photograph archive. Importantly, no visual observations were undertaken of covered cadavers to prevent the prolonged removal of the plastic tarp that would have been required for in-situ analysis. The daily ARF photographer only removed the plastic tarp for a short duration to facilitate photography of the cadavers. The plastic tarp was secured by bricks around the cadavers allocated plot and was only in contact with the supine or prone surface of the cadaver. This limited any potential disturbance during removal of the plastic tarp. The ARF takes photographs of cadavers in an anatomically standard order of sequence, from 
the date of placement to skeletonization [30]. The inter-observer reliability of the TBS scoring method has previously been researched finding high concordance rates between assessors [31,32]. This has also been confirmed in the high inter-observer reliability rates when comparing digital images with in-situ observations, which supports the use of photographs as a suitable proxy for taphonomic observations in the field $[33,34]$.

\section{Point-Scoring Decomposition}

The Megyesi et al [13] method was used to score the decomposition state of the head, trunk and limbs of 26 cadavers each day, for 7 days. The points were then summed to obtain a TBS between 3 (fresh) and 35 (dry bone). The TBS was then inputted into the linear regression formula to provide an estimated ADD (Table 2).

\begin{tabular}{cc}
\hline Megyesi et al [13] & ADD $=10^{\left(0.002^{*} \mathrm{TBS}^{*} \mathrm{TBS}+1.81\right)} \pm 388.16$ \\
\hline Moffat et al [16] & TBSsurf $^{1.6}=125 \times \log _{10} \mathrm{ADD}-212$ \\
& $($ ADD calculated from inverse predictions \\
& Table 1 [16]) \\
\hline
\end{tabular}

\section{Table 2 Linear Regression Formula}

The actual ADD for each known PMI day of each case, was then calculated from an on-site daily temperature logger at the ARF using the following standard formula [13]:

\section{$A D D=\underline{\text { Maximum }+ \text { Minimum air temperature }}$ \\ 2}

One single degree day is defined as one entire day where the temperature stays above the threshold by $1^{\circ} \mathrm{C}$. For decomposition onset, this threshold is generally considered to be $0^{\circ} \mathrm{C}$, as decomposition is thought to cease below freezing temperatures [13]. Actual ADD was calculated by summing the average daily temperatures (above $0^{\circ} \mathrm{C}$ ) from the date of cadaver observation (equivalent to the 'date of discovery' in forensic practice), working backwards until the estimated PMI day was reached: defined as the day at which the accumulated ADD sum equaled the estimated ADD value (Table 2) [13]. If at any given PMI day this equalization was not reached by the first date of placement (i.e. PMI day 1), then temperature data for preplacement days were accessed, until the accumulated ADD sum equaled the estimated ADD value. This was Megyesi et al [13] recommendation for calculating ADD in forensic practice. Humidity was also recorded using an on-site data logger.

The 26 cadavers were then re-scored using the Moffatt et al [16] revision of the Megyesi et al [13] formula (Table 2) to compare the accuracy of PMl estimations. The scoring methodology is the same, except for 'TBSsurf', which was used to denote surface depositions, and the re-adjustment of the points-based decomposition scale from 0 (fresh) to 32 (dry bone), to enable a score of zero for 'no decomposition' [16]. Moffat et al [16] do not recommend rearrangement of their equation to find an estimated ADD. Estimated ADD was therefore calculated using their inverse prediction table. 
An associated written record was also kept for each case to note the observable decomposition characteristics present each day. Previously established reference guides containing over 150 photographs of decomposition traits were used as supporting visual references to record the onset of individual decomposition characteristics observed daily, for the duration of the study $[35,36]$.

\section{Statistical Analysis}

Linear regression formulae from the original and revised ADD/TBS methods were applied to the first 7 PMI days for all 26 cadavers (Table 2). XLSTAT software was used in EXCEL to conduct all statistical-based analyses. Linear regression was used to model the relationship between: i) TBS and ADD and ii) TBSsurf and $A D D$, with the predicted values from the algorithms plotted against the actual ADD and known PMI values for each cadaver. Using adjusted $\mathrm{R}$ square values, the relationship was further delineated to produce equations that best predicted the accumulated level of decomposition (as measured by TBS and TBSsurf) from the known PMI, within the 95\% confidence prediction interval. Two sample t-tests were used to determine if the TBS/TBSsurf methods were accurate measures of known ADD and PMI values, with statistical significance considered at $p<0.05$.

To assess the effect of seasonality (determined by the different months of placement) on the accumulated decomposition (measured by the average TBS at PMI day 7), a two-sample Kolmogorov-Smirnov (K-S) test was used. While decomposition is considered a continuous process, the TBS/TBSsurf scores generated represent ordinal data and should arguably be treated as such when subject to statistical analysis. The K-S test is a non-parametric test suitable for assessing if two independent samples of ordinal data are from the same distribution; irrespective of sample size [37]. For the purpose of this study, the K-S test lends itself to unbiasedly determine if the maximum absolute difference in the accumulated decomposition scores of two samples (e.g. January and April) are significant (derived from different distributions), or insignificant (follow the same distribution). The K-S test generates a $D$ statistic that is the maximum deviation between the two samples tested. The higher the $D$ value, the greater the variance between two samples, with statistical significance considered at $\mathrm{p} \leq 0.05$. All season and month combinations were compared.

\section{Results}

\section{Sample Observations of Decomposition Characteristics}

Using the supporting visual decomposition reference guides [35, 36], a combined total of 18 decomposition characteristics were observed on the 26 human cadavers over the first 7 days post-placement (Figure 1). 


\begin{tabular}{|c|c|c|c|c|c|c|c|c|}
\hline & \multirow{2}{*}{\begin{tabular}{|l|} 
Key \\
$n=$ number of cadavers \\
\end{tabular}} & \multicolumn{7}{|c|}{ PMI (Days) } \\
\hline & & Day 1 & Day 2 & Day 3 & Day 4 & Day 5 & Day 6 & Day 7 \\
\hline \multirow{18}{*}{ 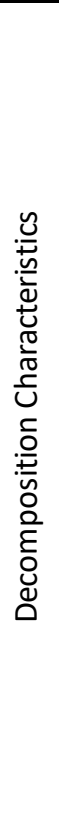 } & Livor Mortis & 23 & 25 & 19 & 14 & 5 & 4 & 4 \\
\hline & Adult Blow Flies & 14 & 15 & 18 & 16 & 11 & 14 & 9 \\
\hline & Skin Slippage & 7 & 9 & 13 & 17 & 17 & 16 & 16 \\
\hline & Abdominal Green Discoloration & 6 & 7 & 11 & 14 & 14 & 11 & 10 \\
\hline & Oviposition & 5 & 14 & 20 & 19 & 15 & 12 & 9 \\
\hline & Marbling & 5 & 4 & 8 & 9 & 15 & 13 & 11 \\
\hline & Blistering & 1 & 4 & 目 & 4 & 5 & 5 & 6 \\
\hline & Tissue Sloughing & 0 & 臬 & 4 & 6 & 10 & 10 & 13 \\
\hline & Bone Exposure & 0 & 百 & 4 & 6 & 7 & 7 & 10 \\
\hline & Scavenging & 0 & 4 & 5 & 8 & 8 & 7 & 3 \\
\hline & Fluid Purge (Facial Orifices) & 0 & h & [2 & 1 & 6 & 5 & 4 \\
\hline & Larvae Activity & 0 & 0 & 11 & 16 & 20 & 20 & 20 \\
\hline & Cadaver Decomposition Island & 0 & 0 & 4 & 4 & 8 & 9 & 11 \\
\hline & Abdominal Bloating & 0 & 0 & $\overline{1}$ & 7 & 9 & 13 & 13 \\
\hline & Brown/Black Discoloration & 0 & 0 & 1 & 10 & 12 & 16 & 17 \\
\hline & Hair Loss & 0 & 0 & 0 & 6 & 10 & 11 & 9 \\
\hline & Mummification & 0 & 0 & 0 & E & B & 4 & 6 \\
\hline & Skin Degloving & 0 & 0 & 0 & 2 & 4 & 8 & 8 \\
\hline
\end{tabular}

Figure 1 Onset of Decomposition Characteristics by PMI (days)

At 1 PMI day, 25 out of the 26 human cadavers displayed one, or more, of the following 7 characteristics of decomposition: 'livor mortis' ( $n=23$ bodies), 'adult blow flies' ( $n=14)$, 'skin slippage' $(n=7)$, 'abdominal green discoloration' ( $n=6)$, 'marbling' ( $n=5)$,'oviposition' (egg laying) $(n=5)$ and 'blistering' ( $n=1)$ (Figure 1). This increased to a total of 18 observable decomposition characteristics recorded by PMI day 7 . The frequency of the decomposition characteristics increased rapidly between 1 to 4 PMI days before reaching a plateau at 5 PMI days. A two-sample t-test demonstrated that there was no significant difference in the number of new decomposition characteristics recorded between 5 PMl days and 7 PMl days $(p<0.05)$, where the decomposition appeared to stabilize (Figure 1).

Early decomposition traits such as 'livor mortis' were resolved in all bodies by 6 PMI days, except for the winter cases that still presented livor mortis at PMI day 6 and PMI day $7(n=4)$. The onset of 'larvae activity' occurred from 3 PMI days $(n=11)$ along with the 'cadaver decomposition island' $(n=4)$ and 'fluid purge' from the facial orifices $(n=2)$. 'Bloating' of the abdomen was observed as early as 4 PMI days in 7 cadavers, along with 'hair loss' $(n=6)$ and 'degloving' of the skin from the hands and/or feet $(n=2)$, which also occurred at day 4. By 6 PMI days an additional 6 cadavers displayed abdominal 'bloating' $(n=13)$. Carnivore scavenging affected 11 cadavers and was present as early as 2 PMI days $(n=4)$ and was quickly associated with 'tissue sloughing' and 'bone exposure' in those body regions exposed to scavenging activity (Figure 1). At PMI day 3 , there were a further 3 new cases of scavenging and by PMI day 4, there were a further 4 new cases of scavenging bringing the total number of cases to 11 (data not shown). Between PMI days 5 to 7 , there were no new cases of scavenging, rather those cadavers were 're-scavenged'. Of the 11 scavenged bodies in the sample, the covering condition had no effect on the presence of scavenging: uncovered control $(n=6 ; 46 \%)$, covered in black plastic tarp $(n=2 ; 33 \%)$ and caged $(n=3 ; 42 \%)(p>0.05)$; where the percentages represent the proportion of scavenged cadavers within each condition. No cadavers presented 'bone exposure' when scavenging was absent. 


\section{Seasonality}

Spring cadavers $(n=5)$ displayed a combined total of 15 decomposition characteristics over 7 PMI days and accounted for the slowest average onset of 13 out of 18 decay traits (72\%) compared to the other 3 seasons $(\mathrm{n}=21)(\mathrm{p}<0.05)$ (Figure 2). For example, 'skin slippage' had an average onset of $5.5 \mathrm{PMI}$ days in spring, compared to 2.9 and 1.6 PMI days in summer and autumn, respectively. Furthermore, spring cadavers when compared to winter cadavers, had a slower onset for both 'livor mortis' (2.5 days vs 1.25 days) and 'brown/black skin discoloration' (6.5 days vs 4 days). Spring cadavers did not display 'degloving of skin', the 'cadaver decomposition island' or 'blistering' decomposition characteristics throughout the 7-day period.

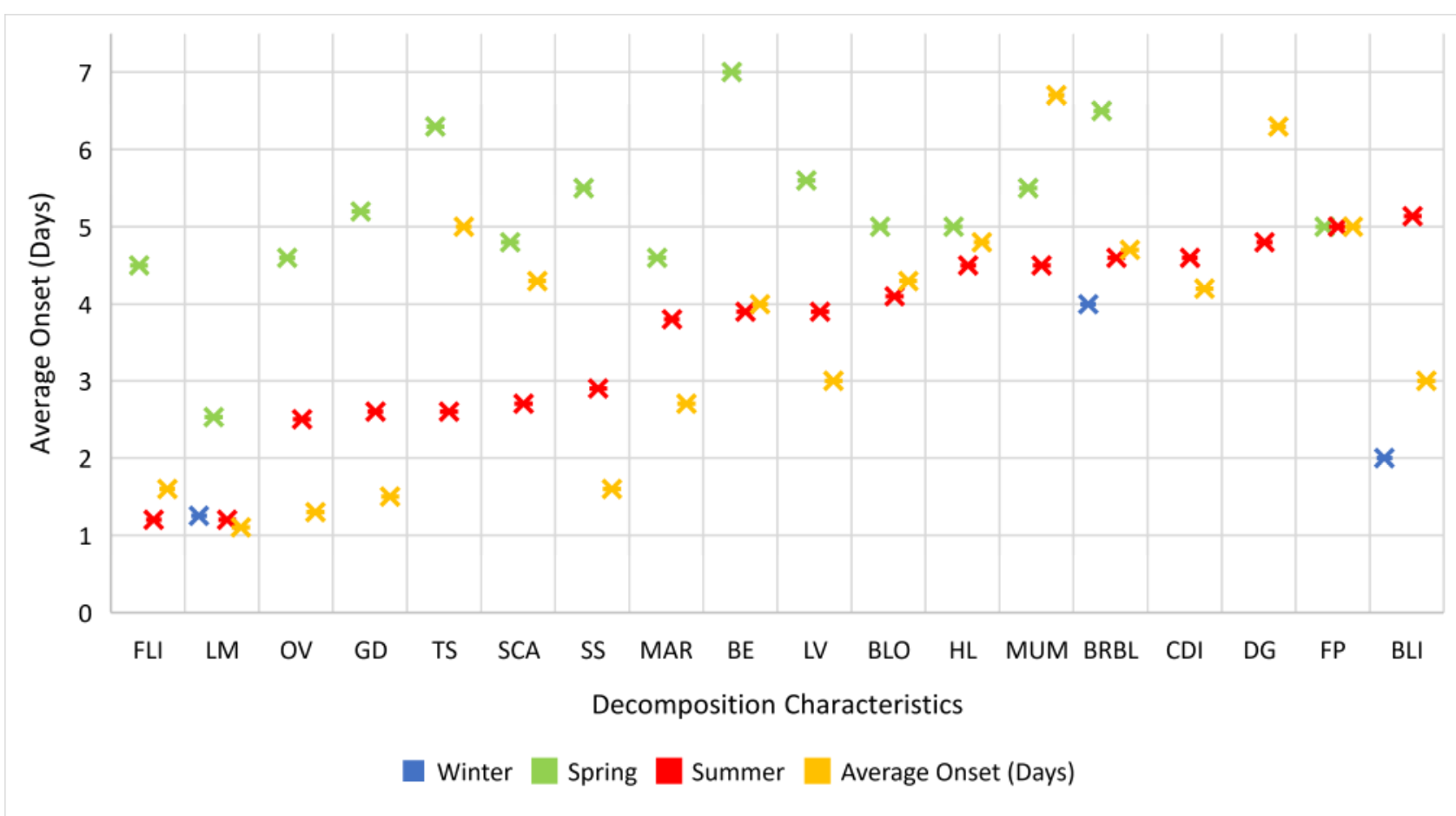

Figure 2. Average Onset (in PMI days) of Decomposition Characteristics by Season

\section{Key of Decomposition Characteristics (from left to right)}

$\mathrm{FLI}=$ Adult blow flies, $\mathrm{LM}=$ Livor Mortis, OV = Oviposition (eggs), GD = Green Discoloration of the Abdomen, TS = Tissue Sloughing, SCA = Scavenging, $S S=$ Skin Slippage, $M A R=$ Marbling, $B E=$ Bone Exposure, $L V=$ Larvae Activity, $\mathrm{BLO}=$ Bloating of the Abdomen, $\mathrm{HL}=$ Hair Loss, $\mathrm{MUM}=$ Mummification, $\mathrm{BRBL}=$ Black/Brown Skin Discoloration, CDI = Cadaver Decomposition Island, DG = Degloving of skin, $\mathrm{BLI}, \mathrm{FP}=$ Fluid Purge, BLI = Blistering.

Summer and autumn cadavers $(n=17)$ displayed 18 decomposition characteristics and had the fastest average onset for 15 decomposition characteristics (83\%) compared to winter and spring trials $(n=9)(p<0.05)$ (Figure 2). Upon closer observation, summer cadavers $(n=10)$ accounted for the fastest average onset of 8 decomposition characteristics, and autumn cadavers $(n=7)$ had the fastest average onset for 7 decay traits. There was no statistical difference in the average onset (in PMI days) of decomposition characteristics displayed between summer and autumn cadavers $(p>0.05)$. Autumn cadavers $(n=7)$ had the slowest average onset for one decomposition characteristic: 'desiccation of skin' at 6.7 PMI days. Winter cadavers $(n=4)$ only displayed 3 decomposition characteristics over 7 PMI days and had the fastest average onset for 'blistering' (2 PMI days) and 'brown/black skin discoloration' (4 PMI days) compared to the other seasons ( $\mathrm{n}=22$ ). 'Fluid purge' was the only decomposition characteristic that was recorded as having the same average onset of 5 
PMI days for spring, summer and autumn cadavers $(n=22)$. Summer cadavers had the fastest onset for 'scavenging' at 2.6 PMI days, whereas spring cadavers had the slowest onset for this trait at 4.8 PMI days. Early-onset scavenging correlated with the onset of high-TBS advanced decomposition characteristics such as 'tissue sloughing' and 'bone exposure', which were most prominent in summer cadavers.

\section{$\underline{\text { TBS/TBSsurf Scores }}$}

Using the Megeysi et al [13] TBS point-scoring method, it was found that the assigned TBS ranged from 3 (fresh decomposition) to 26 (skeletonization) for this dataset. The highest possible score of the TBS method is 35, which was not represented in this dataset. At PMI Day 1,50\% of the sample scored within a TBS of 3 , while the remaining 13 cadavers were assigned 'early' decomposition TBS scores (>3 $\leq 7)$. By 7 PMI days, 15 cadavers, (58\% of the total sample) scored within the 'advanced decomposition' category in at least one or more of the three classification regions: i) Head and Neck (TBS $\geq 7$ ), ii) Trunk (TBS $\geq 6$ ) and iii) Limb (TBS 26). Conversely, the TBSsurf scores ranged between 3 (fresh decomposition) and 23 (skeletonization) for the dataset and were significantly lower than TBS scores at each PMI day $(p<0.05)$. This was expected when considering that a TBS of 3 is proportionally equal to a TBSsurf of 0 [16]. The average TBS and TBSsurf scores assigned each day to the 26 cadavers represent a curvilinear relationship with the PMI as shown in Figure 3.

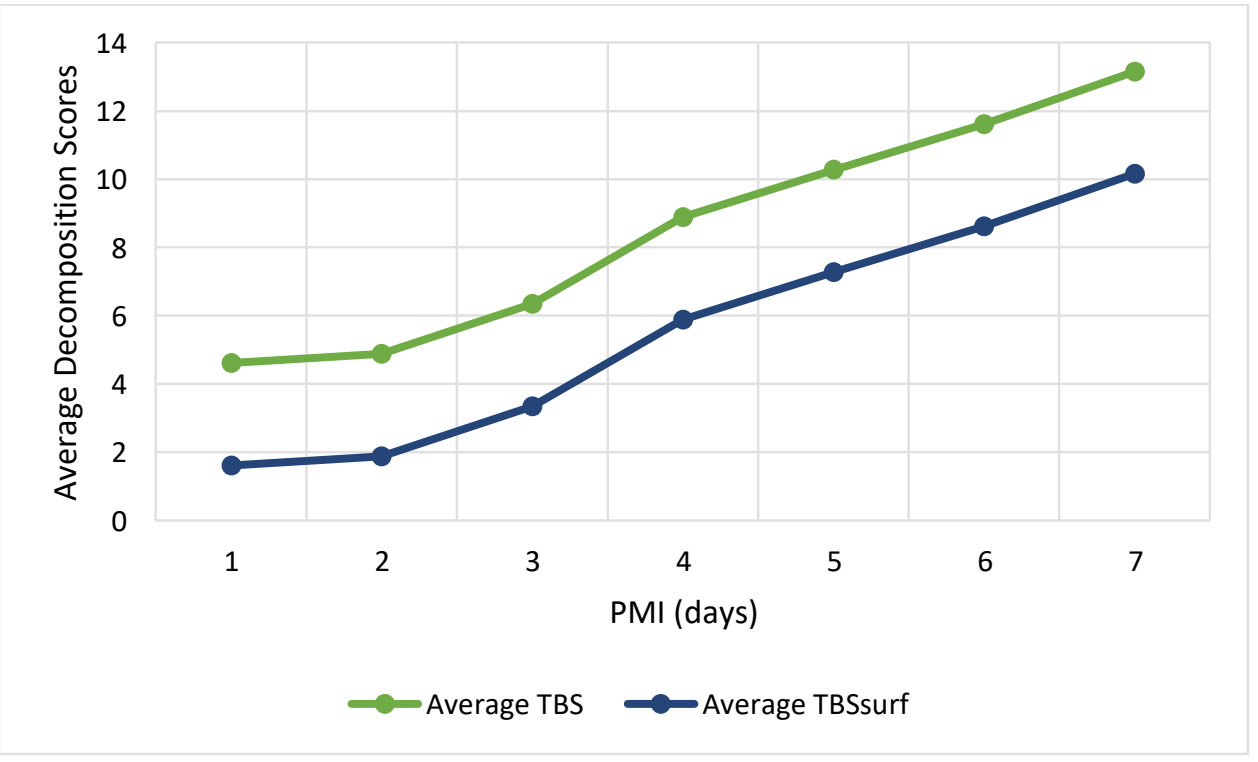

\section{Figure 3. Average TBS and TBSsurf Decomposition Scores in the First 7 Days Post-Death ( $n=26$ cadavers)}

\section{Seasonality and TBS/TBSsurf}

Winter cadavers $(n=4)$ had the lowest average TBS from 1 to 7 PMI days compared to all other seasons $(p<0.05)$. The winter cadavers had a constant average TBS of 3 for the first 3 PMI days, which then increased to an average of 4.3 TBS between 4 - 7 PMI days (Figure 4). 


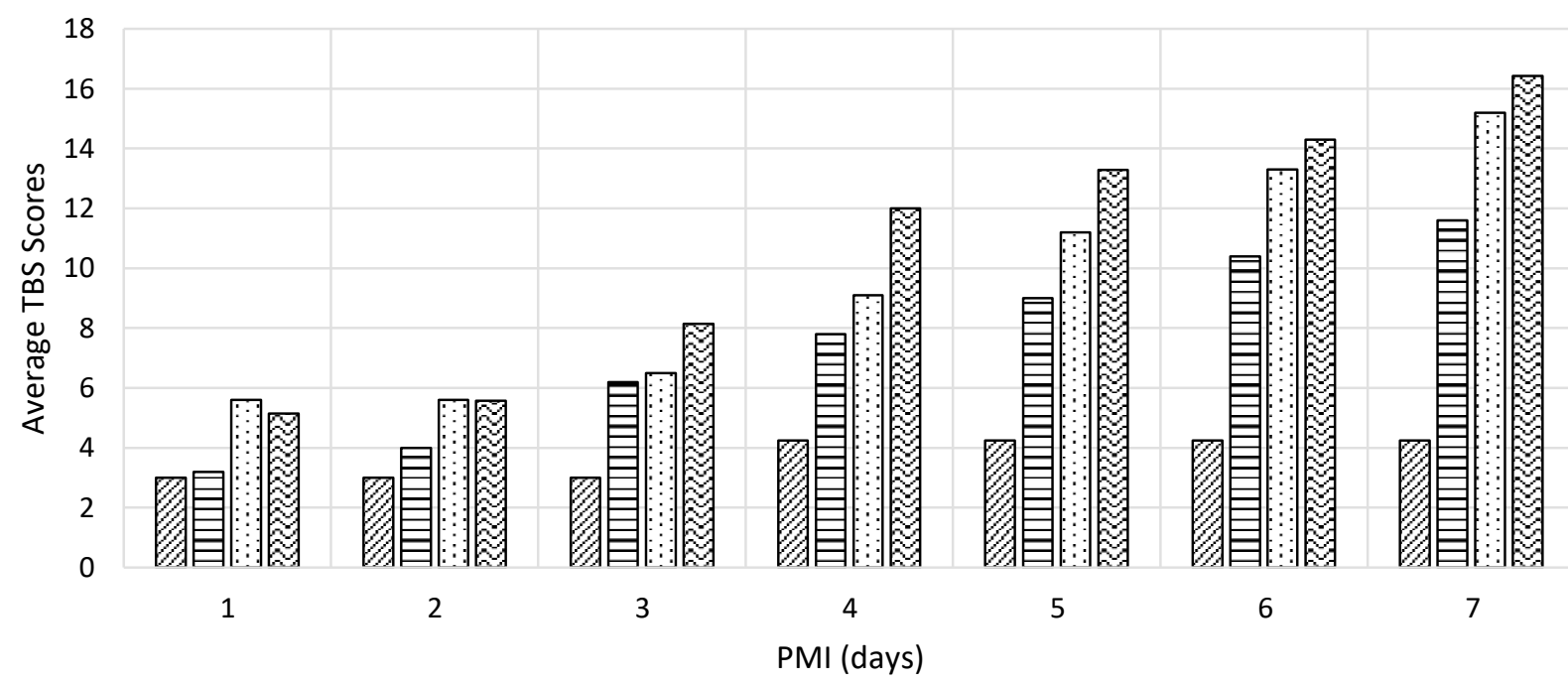

口Winter $\boxminus$ Spring $\boxminus$ Summer 因Autumn

Figure 4 Distribution of Average TBS Scores (PMI Days 1-7) by Season

For spring, summer, and autumn cadavers, the average TBS increased with each PMI day up to 7 days, except for PMI days 1 and 2 in the summer cadavers $(n=10)$, where no change in the average TBS was reported. Spring cadavers $(n=5)$ had a significantly lower TBS average than summer $(n=10)$ and autumn $(n=7)$ cadavers $(p<0.05)$. Summer cadavers had the highest average TBS for PMI days 1 and 2, whereas autumn cadavers had the highest average TBS between PMI days 3 to 7 . Between PMI days 1 to 3 there was no statistical difference between autumn and summer TBS ( $p>0.05)$, whereas between PMI days 4 to 7 , TBS was statistically higher for autumn cadavers compared to summer cadavers $(p<0.05)$ (Figure 4). Comparable results were found for TBSsurf scores (not shown).

To further assess the effect of seasonality on the accumulated decomposition a non-parametric two sample Kolmogorov-Smirnov (K-S) test was used to compare the distribution of actual TBS scores over 1-7 PMI days between the months of cadaver placement. This resulted in 28 possible combinations of month comparisons (Table 3).

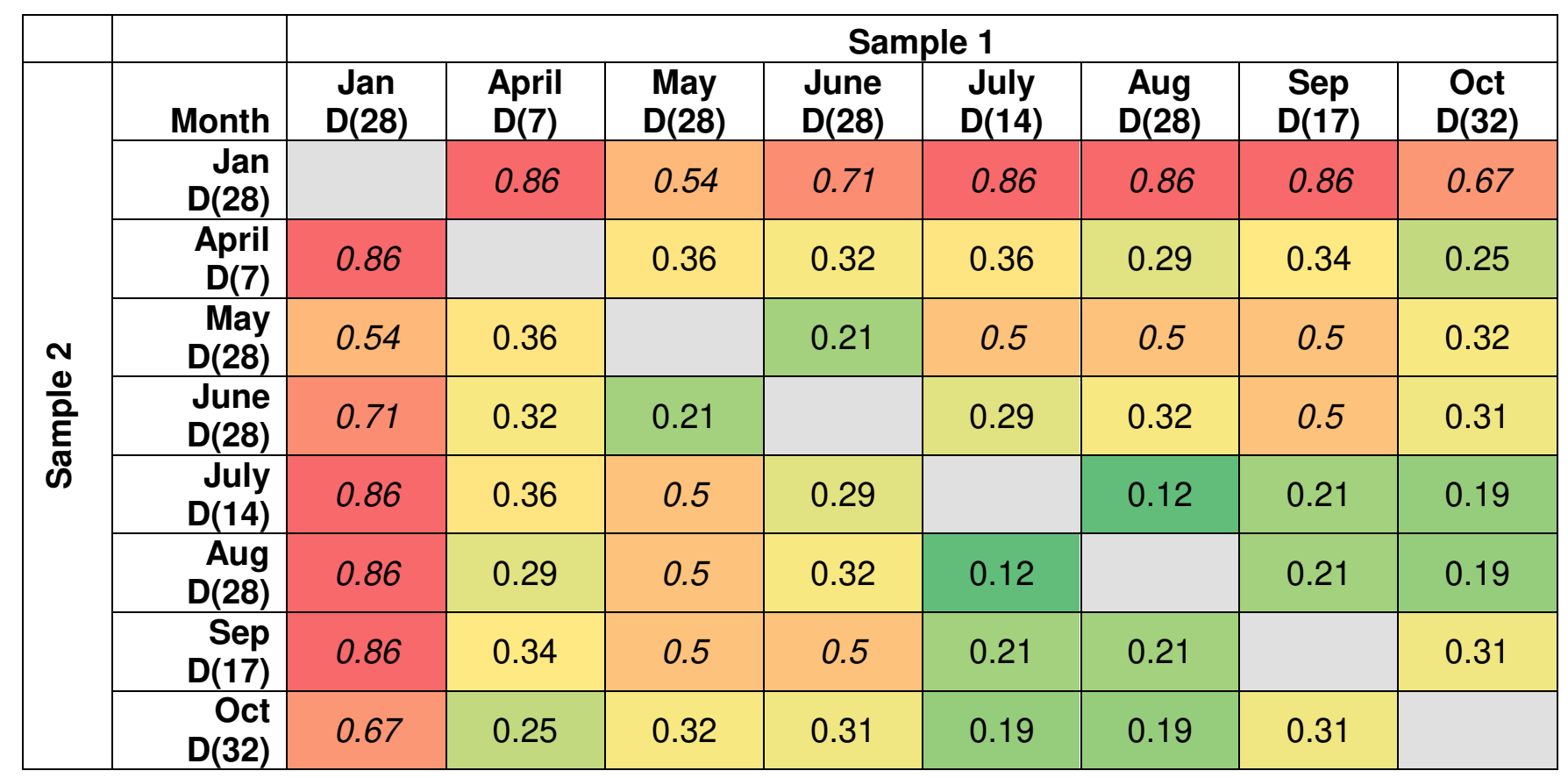


Table 3 Kolmogorov-Smirnov Results: Heat Map where $D(n)$ is the total frequency of TBS recorded over the 7day period for the placement month. For example, in January, 4 bodies were observed daily over 7 days, which generated a TBS of 28 hence $\mathrm{D}(28)$, whereas in April, 1 body was observed daily over 7 days, which generated a TBS of 7 , hence $D(7)$. Therefore, the frequency of TBS for the comparison of January (Sample 1) and April (Sample 2) is $D(35)$. The value of the $D$ statistic is shown in boxes on the heat map where: red = high variance between the two samples, and green = low variance between the two samples. The higher the $\mathrm{D}$ statistic the greater the variance between the two samples. Statistically significant results are denoted by italics on heat map $(p \leq 0.05)$.

The K-S results indicated that the TBS from the January placements deviated significantly from the TBS of decomposing cadavers of all other months $(p \leq 0.05)$ (Table 3$)$. When the TBS was compared between months of the same season (for example the summer months of June/July, July/August and June/August) there was no significant deviation between the scores $(p \geq 0.05)$. This suggests that TBS was normally distributed within each season. When comparing the TBS of the autumn month (October) to spring months (April and May) and summer months (June, July and August), there were no significant deviations between the scores $(p \geq 0.05)$. This indicates that October's TBS followed the same distribution as spring and summer TBS. Similarly, TBS for the spring month of April found statistically insignificant variance when compared to summer months (June, July and August) and Autumn (September and October) $(p \geq 0.05)$. This suggests that spring TBS was similarly distributed with Autumn and Summer TBS.

\section{Temperature}

The average daily temperature data from ARF onsite records was used to calculate ADD values for each known PMI day using the TBS [13] and TBSsurf [16] methods. Table 4 depicts seasonal components such as the average temperature, humidity and rainfall for the month of placement, to demonstrate climatic conditions cadavers were exposed to. It also includes the estimated ADD values that were derived by inputting the TBS and TBSsurf scores into the equations in Table 2 and averaged over the study's duration (1-7 PMI days) (Table 4).

\begin{tabular}{ccccccc}
\hline Month & $\begin{array}{c}\text { Average } \\
\text { Temperature }{ }^{\circ} \mathrm{C} \\
(\text { High } / \mathrm{Low})\end{array}$ & $\begin{array}{c}\text { Average } \\
\text { relative } \\
\text { Humidity } \\
(\%)\end{array}$ & $\begin{array}{c}\text { Average } \\
\text { Rainfall } \\
(\mathrm{mm})\end{array}$ & $\begin{array}{c}\text { Average } \\
\text { TBS- } \\
\text { Estimated } \\
\text { ADD }\end{array}$ & $\begin{array}{c}\text { Average } \\
\text { TBSsurf- } \\
\text { Estimated } \\
\text { ADD }\end{array}$ & $\begin{array}{c}\text { Average } \\
\text { Actual } \\
\text { ADD }\end{array}$ \\
\hline January & $\begin{array}{c}3^{\circ} \mathrm{C} \\
\left(17^{\circ} \mathrm{C} /-14^{\circ} \mathrm{C}\right)\end{array}$ & 66 & 3.6 & 70 & 51 & 6 \\
\hline April & $\begin{array}{c}16^{\circ} \mathrm{C} \\
\left(29^{\circ} \mathrm{C} / 1^{\circ} \mathrm{C}\right)\end{array}$ & 66 & 3.9 & 100 & 73 & 15 \\
\hline May & $\begin{array}{c}21^{\circ} \mathrm{C} \\
\left(32^{\circ} \mathrm{C}-6^{\circ} \mathrm{C}\right)\end{array}$ & 65 & 1.9 & 93 & 68 & 19 \\
\hline June & $\begin{array}{c}25^{\circ} \mathrm{C} \\
\left(36^{\circ} \mathrm{C}-15^{\circ} \mathrm{C}\right)\end{array}$ & 70 & 5.4 & 108 & 78 & 26 \\
\hline July & $\begin{array}{c}25^{\circ} \mathrm{C} \\
\left(33^{\circ} \mathrm{C} / 19^{\circ} \mathrm{C}\right)\end{array}$ & 75 & 6.3 & 133 & 95 & 26 \\
\hline August & $\begin{array}{c}24^{\circ} \mathrm{C} \\
\left(34^{\circ} \mathrm{C} / 16^{\circ} \mathrm{C}\right)\end{array}$ & 72 & 3.3 & 128 & 92 & 25 \\
\hline September & $\begin{array}{c}22^{\circ} \mathrm{C} \\
\left(33^{\circ} \mathrm{C} / 8^{\circ} \mathrm{C}\right)\end{array}$ & 73 & 1.4 & 325 & 199 & 21 \\
\hline October & $\begin{array}{c}16^{\circ} \mathrm{C} \\
\left(27^{\circ} \mathrm{C} / 2^{\circ} \mathrm{C}\right)\end{array}$ & 73 & 2.9 & 116 & 84 & 12 \\
\hline
\end{tabular}

Table 4. Average monthly temperatures (taken from ARF records) and average estimated and actual ADD values over 1-7 PMI Days 
The average estimated ADD values from both the TBS and TBSsurf methods were significantly overestimated when compared to the actual ADD values for each month of cadaver placement using a twosample t-test $(p<0.05)$. For 3 of the 4 cadavers placed in January, the estimated ADD remained stagnant at 70 ADD (TBS) and 51 ADD (TBSsurf) for the duration of 1-7 PMI days and was statistically different to the actual ADD, which ranged from 0 ADD (day 1 and 2) to 8 ADD (day 7$)(p<0.05)$. Conversely, for the summer months the estimated ADD (from TBS) ranged from 67 ADD (day 1) to 407 ADD (day 7) for June cadavers $(n=4)$ and 76 ADD (day 1) to 407 ADD (day 7$)$ for July and August placements $(n=6)$.

From Table 4 it is evident that the actual ADD values correlate positively with the average monthly temperature. For example, 6 was the lowest actual ADD value (averaged over PMI days 1 - 7) and this corresponded to a lowest average monthly temperature in January of $3^{\circ} \mathrm{C}$. Conversely, the highest actual ADD value was 26 (averaged over PMI days 1 - 7) corresponded to the highest average monthly temperature in both June and July at $25^{\circ} \mathrm{C}$. The average relative humidity ranged from $66-75 \%$ for the placement months and was significantly higher for summer and autumn months than spring and winter months $(p<0.05$; twosample t-test). Summer months had the highest average rainfall $(5 \mathrm{~mm})$, whereas the autumn season had the lowest average rainfall $(2.15 \mathrm{~mm})$, albeit this difference was insignificant $(p>0.05)$

\section{The Effect of Body Coverings}

The varying covering conditions appeared to have no effect on the level of accumulated decomposition as reported by insignificant differences between the covering condition and the TBS at each PMI day per seasonal trial ( $p>0.05)$. By PMI day 7 , the control (uncovered) cadavers reached a slightly higher average TBS than covered or caged cadavers across all seasons except spring; where the average TBS was equal between the control (uncovered) and covered cadavers (Figure 5). Although summer cadavers showed the greatest difference in the average TBS between control (uncovered) and covered cadavers, this was by a marginal difference of 4 TBS and was statistically insignificant $(p>0.05)$.

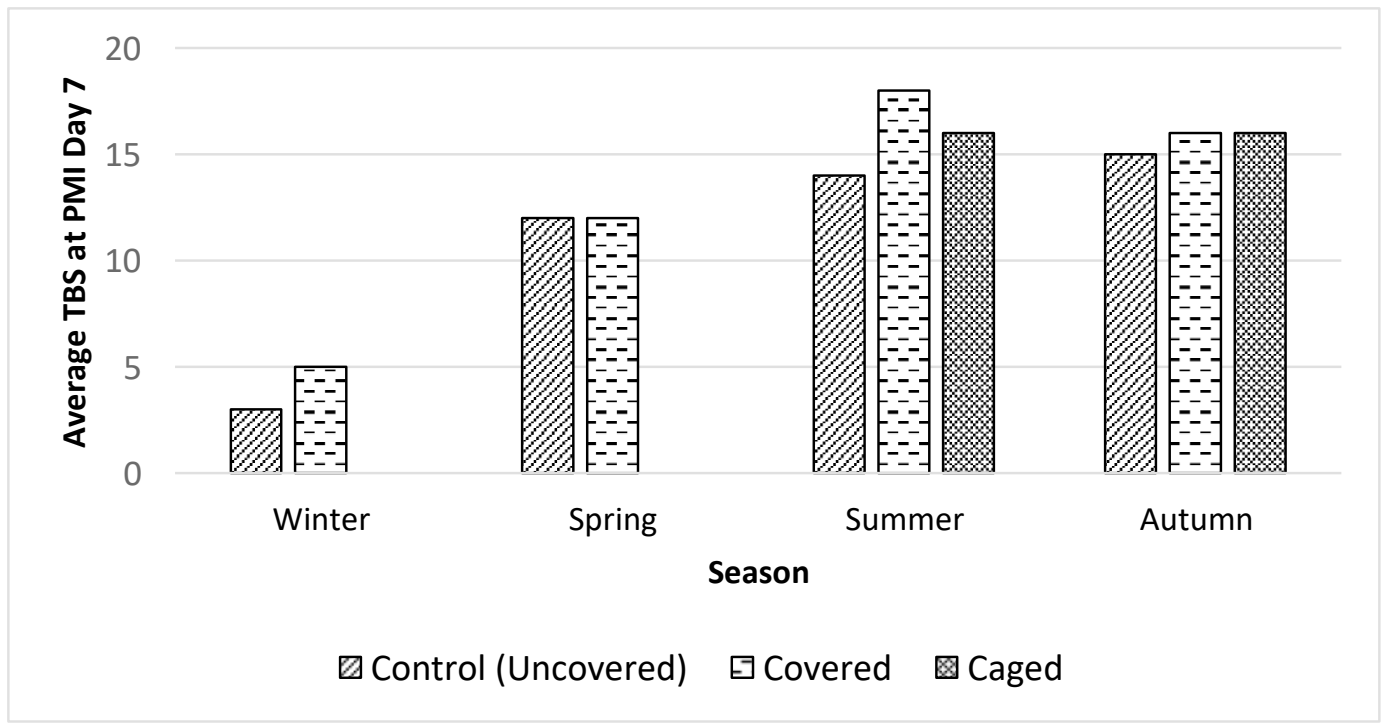

Figure 5 The Effect of Coverings on the average TBS at PMI Day 7 by Season 
Linear regression analysis was used to demonstrate the relationship between: i) the level of accumulated decomposition (as measured by TBS and TBSsurf), ii) estimated and actual ADD, and iii) the estimated and known PMI.

\section{TBS/TBSsurf and ADD}

Regression of the dependent variable TBS by the estimated ADD (independent variable) produced a curvilinear relationship with an $\mathrm{R}^{2}$ value of 0.5 , which improved to 0.9 when the $\mathrm{x}$ axis was log-transformed (Figure 5). This indicates that $90 \%$ of the variability in the decomposition scores in this study could be attributed to accumulated temperature. However, regression of TBS by the actual ADD values calculated for the first $7 \mathrm{PMl}$ days for each cadaver, obtained an $\mathrm{R}^{2}$ value of 0.025 indicating no existing relationship between the actual ADD and the level of decay (Figure 6).

Figure 5 Log-transformed estimated ADD vs TBS ( $n=26)$

Figure 6 Actual ADD vs TBS $(n=26)$
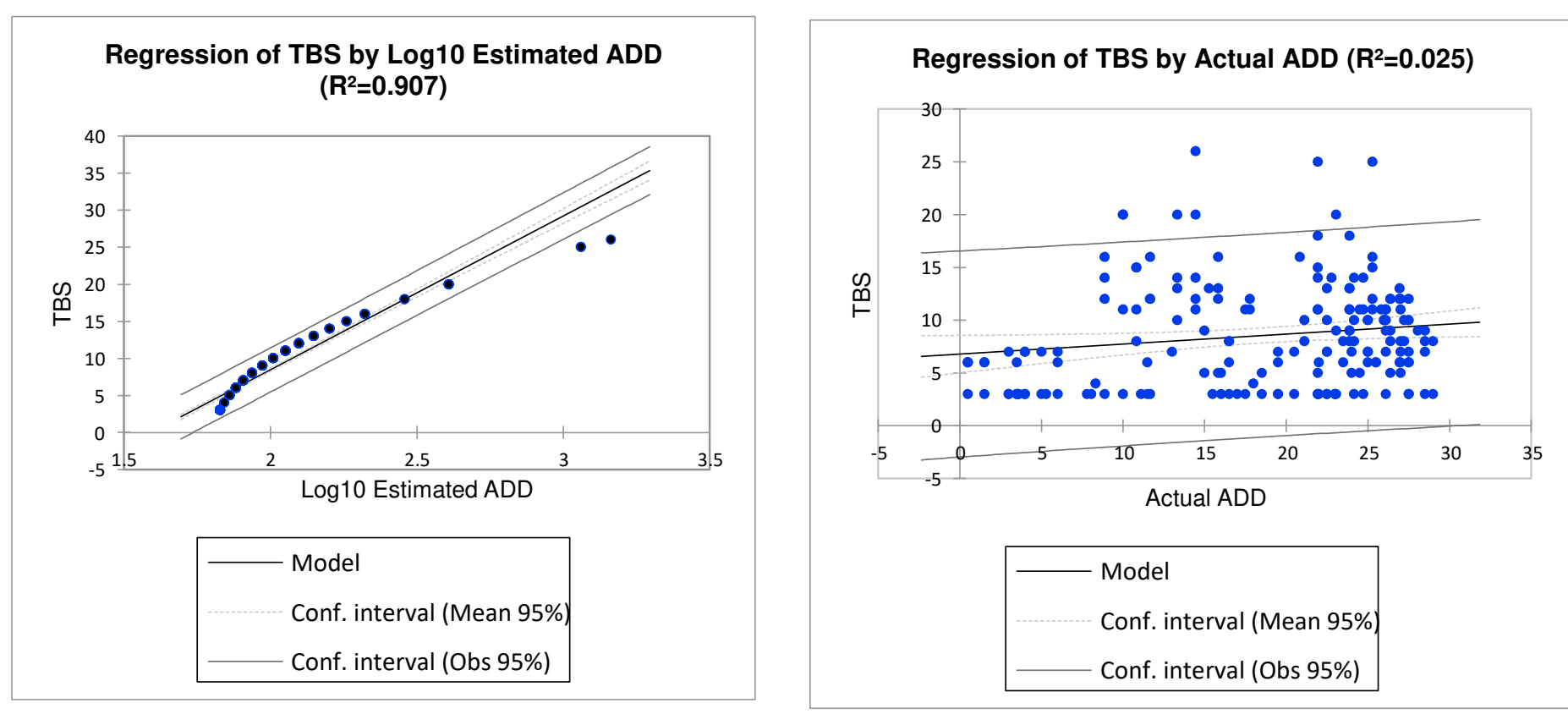

Regression of seasonal TBS scores for all seasons (except winter), by actual ADD values had comparably low $R^{2}$ values $(\leq 0.025)$ (results not shown). This was further supported by a paired t-test that revealed a statistically significant difference between the estimated ADD values (derived from the Megyesi et al [13] equation) and the actual ADD for each season $(p<0.05)$. However, regression of winter cadaver TBS scores by actual $A D D$, improved the $R^{2}$ value to 0.57 .

Similarly, using the Moffat et al [16] revision of the Megyesi et al [13] ADD formula (Table 2), it was found that regression of TBSsurf by the estimated ADD produced a curvilinear relationship and a slightly improved $R^{2}$ value of 0.58 , which further increased to an $R^{2}$ of 0.93 when the estimated ADD was log-transformed. When actual $A D D$ values were inputted, $R^{2}$ was reduced dramatically to 0.13 implying that there is no relationship between the two variables of TBSsurf and actual ADD. This was also corroborated by a paired t-test showing significant differences between the estimated ADD values (using the TBSsurf method [16]) and the actual ADD at each PMI day ( $p>0.05)$. 


\section{$\underline{\text { TBS/TBSsurf and PMI }}$}

Linear Regression of both TBS and TBSsurf by the known PMl found an $\mathrm{R}^{2}$ value of 0.4 . This suggests that approximately $40 \%$ of the variation in decomposition could be explained by the PMI. The overall model fit improved to $R^{2}=0.65$ when only summer TBS scores were included as the response variable. Autumn and spring TBS scores both had an $\mathrm{R}^{2}$ value of 0.55 when the linear regression was completed against the actual PMI, whereas winter TBS scores had the lowest $R^{2}$ value of 0.09 , inferring that almost no variation in the accumulated decomposition could be explained by the PMI.

Two sample t-tests revealed that the estimated PMI (derived from the TBS method) was significantly different from the known PMI, at each PMI day (1-7) $(p<0.05)$. A significant difference was also present between the estimated PMI (from the TBSsurf method) and the known PMI for the first 4 PMI days $(p<0.05)$. However, between PMI days 5 to 7, there was no significant difference between the TBSsurf-estimated PMI and the known PMI ( $p>0.05)$. A box and whisker plot visualizing the range of estimated PMls against the known PMls (days 1-7) shows that both TBS and TBSsurf methods over-estimated the PMI (Figure 7). The upper quartile range derived from TBS and TBSsurf-estimated PMI was 16 days and 13 days respectively, compared to an upper quartile range of 7 days for known PMI $(p<0.05)$. High variability was present above the upper quartile ranges (indicated by dots in Figure 7) with the highest PMI estimated as 24 days (using TBS) and 17 days (TBSsurf) $(p<0.05)$. The median TBSsurf-predicted PMI was equivalent to the median actual PMI at 4 days. Estimated PMls $>25$ days were determined as extreme outliers and removed from the plot (not shown). TBSestimated PMI had the highest error of 62 estimated PMI days and TBSsurf-estimated PMI had the highest error of 36 estimated PMI days, compared to a known PMI of 7 days for a September cadaver (results not shown). 


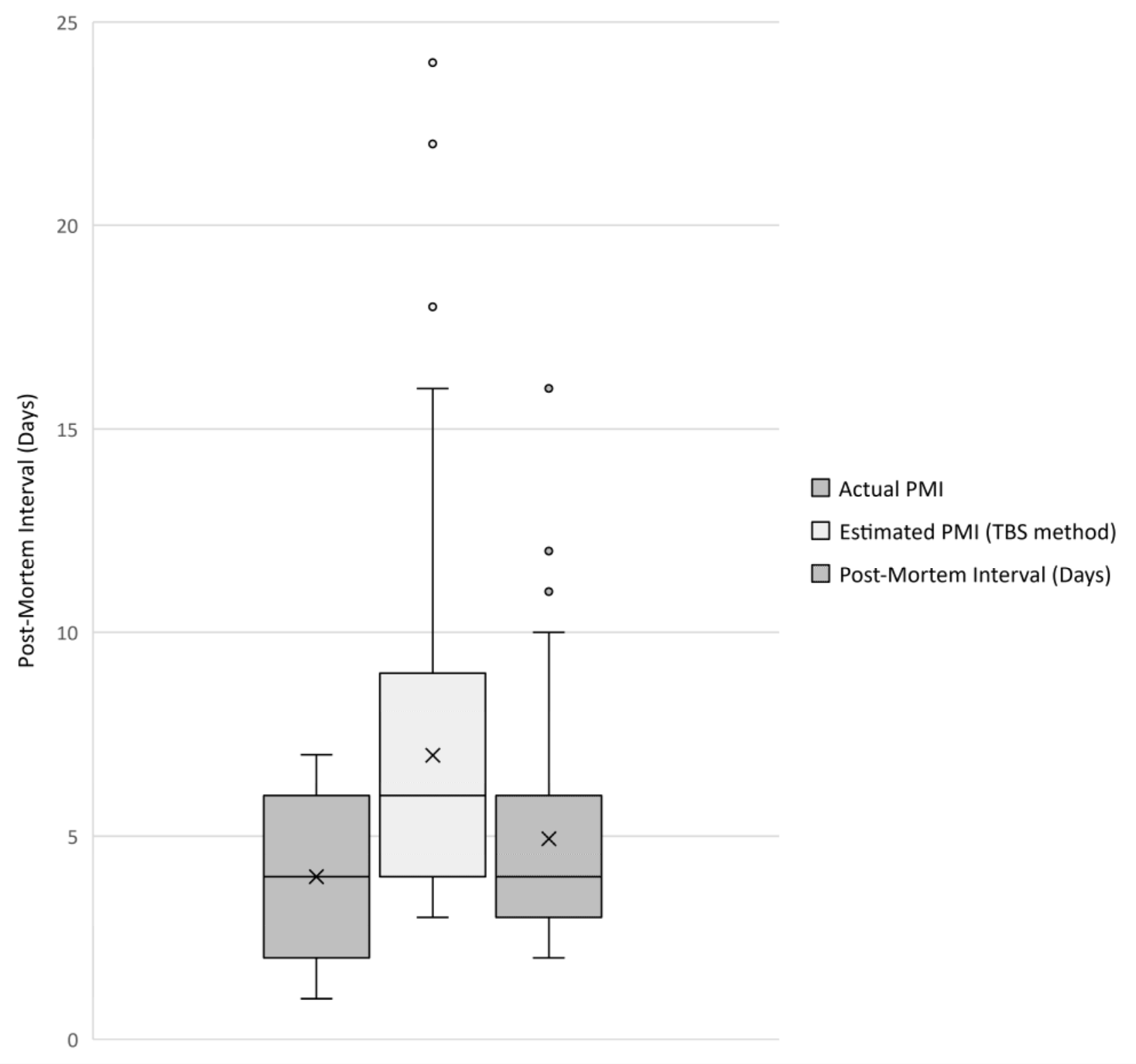

Figure 7. Box and Whisker Plot by PMI ( $n=26)$.

Comparison of the Estimated PMI using the Megyesi et al [13] (orange) and Moffatt et al [16] formulas (grey) to the actual PMI (blue). Values above the upper quartile range are represented by dots. The $x$ within the box plots represent the mean PMI (days). Extreme outliers (defined as >25 PMI days) are not displayed. 
When comparing the estimated PMI from TBS and TBSsurf methods with the known PMI per season, box and whisker plots are also presented (Figure 8).
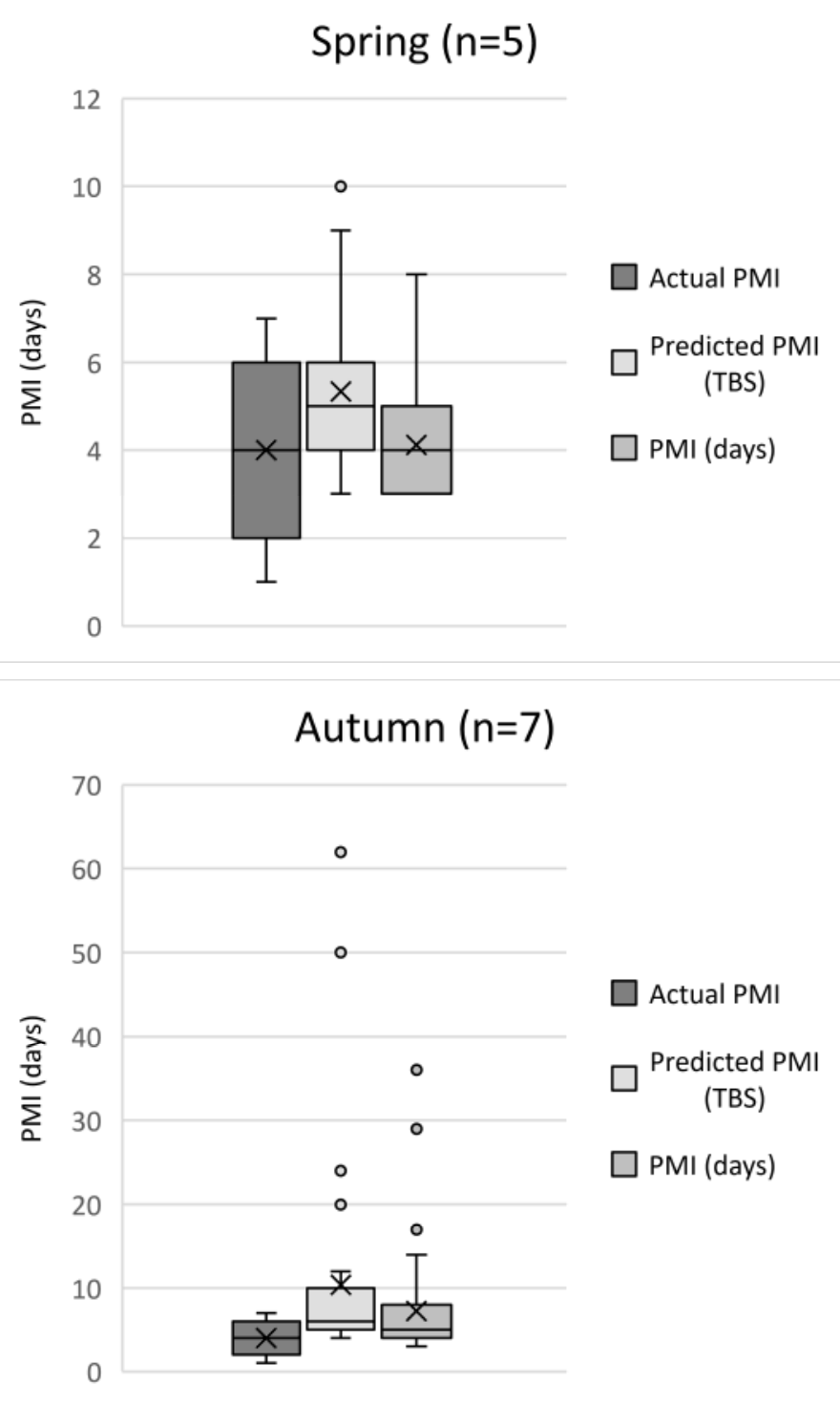
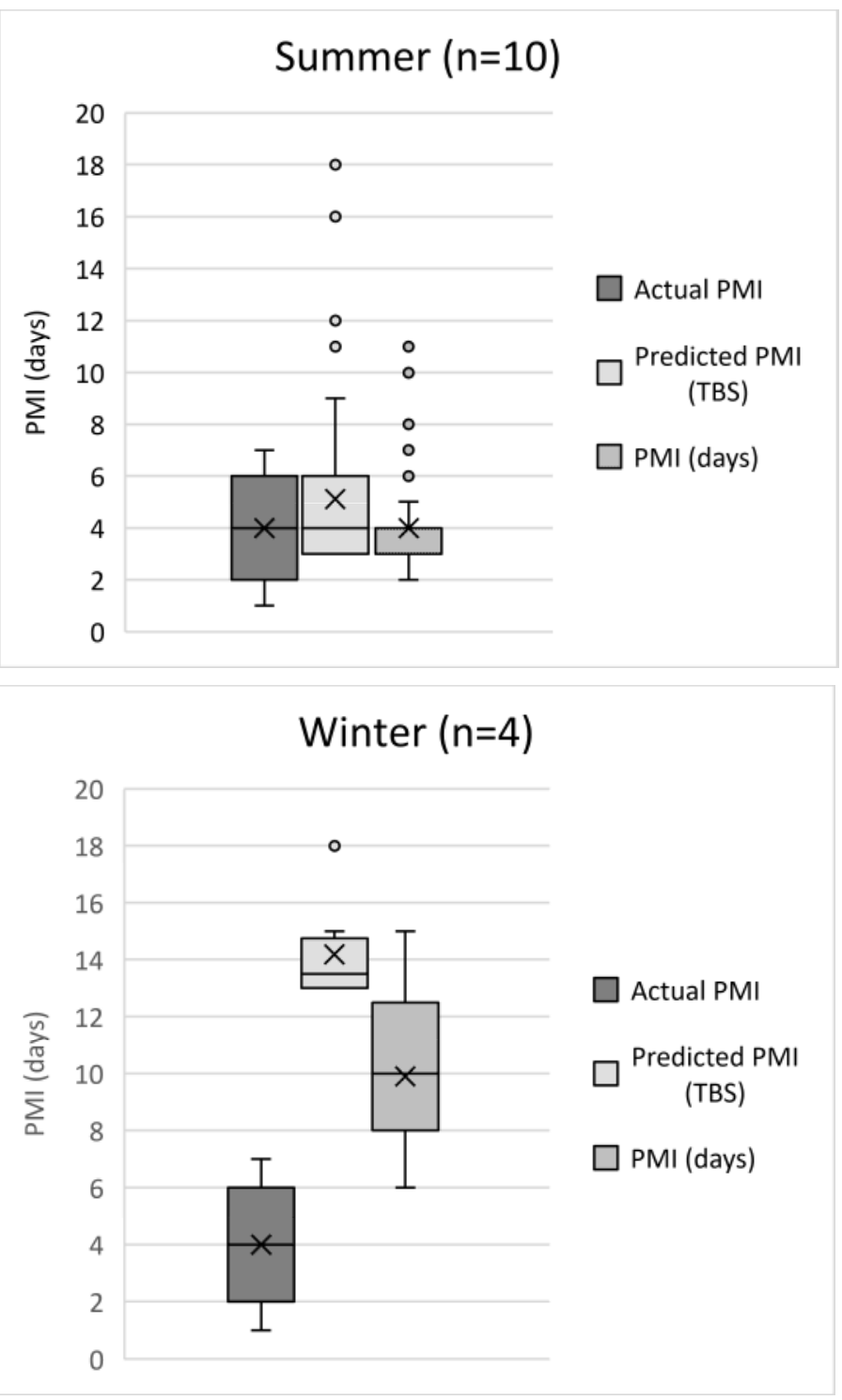

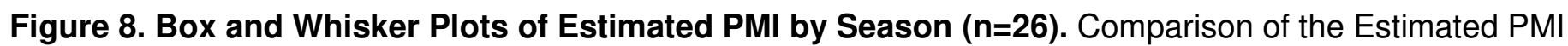
using the Megyesi et al [13] (orange) and Moffatt et al [16] formulas (grey) to the actual PMI (blue) by seasonal cadaver placements. Values above the upper quartile range are represented by dots. The $\mathrm{x}$ within the box plots represent the mean PMI (days).

Overall, spring cadavers $(n=5)$ had the most accurate predictions of TBS and TBSsurf-estimated PMI compared to all other seasons, with only one outlier for the TBS-surf PMI estimation of 10 days (when the known PMI was 7 days) for a May placement. The upper quartile range was equivalent to the known PMI of 6 days for TBS-estimated PMI in both spring and summer cadavers. While summer cadavers $(n=10)$ had the greatest number of combined outliers above the upper quartile range (from TBS and TBSsurf methods) than another other season, the most extreme outliers were represented in the autumn cadavers $(n=7)$. Autumn TBS-estimated PMI had the highest error of 62 estimated PMI days and TBSsurf-estimated PMI 36 days, compared to a known PMI of 7 days, which were both significant over-estimations $(p<0.05)$. For winter cadavers, both TBS and TBSsurf methods also significantly overestimated the PMI. TBS-estimated PMI performed most poorly in winter cadavers with the lowest quartile range of estimated PMI at 13 days, which was 6 days over the upper quartile range of the known PMI at 7 days. 


\section{Discussion}

This paper assessed the effect of seasonality on the relationship between decomposition (quantified by TBS and TBSsurf), temperature (as measured by ADD), and the PMI in the early post-mortem period. Whilst a plethora of forensic taphonomy studies have observed the transition of the body from 'fresh' to 'skeletonization' [15], [18], [19], [23], [25], this paper focused entirely on the application of ADD-based methods to the estimation of the early post-mortem period. This shorter interval frame encompasses the time period where insect larvae activity may be absent (up to 72 hours post-death) and where other experimental techniques such as biochemical markers and physiological changes of PMl estimations are not yet standardised in routine forensic casework [26], [27].

In this study the Megeysi et al [13] TBS model was compared to the Moffat et al [16] TBSsuf method, which are statistically driven systems that seek to estimate the post-mortem interval (PMI) based on calculated ADD values and the observed level of accumulated human decomposition. ADD is the cumulative total of the average daily temperatures and is thought to be the most prominent environmental variable influencing the decomposition process [2]. Linking the accumulated decomposition to ADD allows temperature changes to be accounted for when calculating the PMI and theoretically lends itself suitable to compare seasonal environments, where using the chronology of PMI days alone, does not [23]. To the authors' knowledge this paper was also the first to test the TBSsurf method [16] on 26 human cadavers. In forensic taphonomy, this larger sample size confirms the findings of other studies that have failed to validate the TBS and TBSsurf methods $[21,26]$.

\section{Seasonal Effects on Gross Decomposition}

Decomposition variability is commonly attributed to components of seasonality such as temperature, ADD, humidity and rainfall [2] [11] [17]. However, relatively little research has quantified the effect of seasonality on gross body decomposition [20-21]. This study found that the rate and pattern of decomposition in human cadavers did not vary significantly between all seasons. While spring cadavers had the slowest decay rate over the early post-mortem period, both summer and autumn cadavers accounted for the fastest onset of the majority of decomposition characteristics. The first stage of decomposition in the TBS method is 'fresh' with 'no signs of discoloration' (TBS $\leq 3$ ), which represented spring and winter cadavers at PMI day 1. However, summer and autumn cadavers were assigned scores reflecting 'early' decomposition (TBS $>3-\leq 7$ ) at PMI day 1, with traits such as 'green discoloration of the abdomen' and 'skin slippage' noted, which have previously been found to occur between 2 to 4 PMI days [1] [23]. Given that all cadavers were exposed to the same morgue cooler to equilibrize body temperature pre-placement, this demonstrates that the acceleration of intrinsic decomposition for summer and autumn cadavers was likely dependent on their exposure to a combination of seasonal variables post-placement.

The faster decay rate of summer and autumn cadavers in the early post-mortem period was further corroborated by assignment of comparably higher TBS/TBSsurf at each PMI day compared to winter and spring placements. This was expected for summer cadavers exposed to the highest average seasonal temperatures and actual ADD values, but more surprising for autumn cadavers given their exposure to lower temperature ranges and actual ADD values. Furthermore, the K-S test reported indifferent TBS in October to all summer months despite the significant variation in temperature $\left(9^{\circ} \mathrm{C}\right.$ difference), between these seasons. 
Furthermore, linear regression analysis showed no relationship between accumulated decomposition (as measured by TBS) and the actual ADD for all seasonal placements. Collectively, these results suggest that the effects of temperature appeared negligible to the presentation and trajectory of decomposition characteristics on summer and autumn cadavers during the early post-mortem period.

One possible explanation for the similar decay rate between summer and autumn cadavers is the relative humidity. Research has long suggested that high humidity promotes decomposition by correlating with insect larvae and reducing the onset of mummification [2]. This study also found that insect larvae had the quickest onset in summer and autumn cadavers. Given that summer and autumn cadavers were exposed to a significantly higher average humidity than spring or winter cadavers; this could account for the accelerated decomposition. In addition, the earliest onset of insect larvae occurred from 3 PMI days in autumn cadavers. This aligns with previous findings [8] and provides further justification for the use of this specialist science post-72 hours to estimate the PMI from identification of larval age.

The covering conditions appeared to have no significant influence on the decomposition rate between seasonal trials. However, there were observable differences in the higher average TBS of covered cadavers compared to the caged or control (uncovered) conditions in summer, autumn and winter cadavers. This affirms previous findings that covering of cadavers with plastic tarps accelerates moist decomposition and prolongs insect activity, likely by providing the optimum darker environments for larval activity [39]. Interestingly, the covering condition appeared to have no effect on the occurrence of scavenging, as cadavers were still scavenged in both the covered and caged groups. Racoon scavenging is particularly abundant at the ARF site where this experiment was conducted and can rapidly [40]. Therefore, these preventative measures may not always discourage scavenging activity.

No bodies exhibited mummification in this study, which is considered an advanced stage of decomposition [13]. This is likely due to the shorter post-mortem intervals assessed. However, desiccation (drying) of the skin is considered a precursor for mummification that may occur at any temperature, provided the humidity is high and the environment is dry [12] [41]. 'Desiccation of the skin' had the fastest onset for summer cadavers and slowest onset for autumn cadavers. Since relative humidity was comparable for the summer and autumn trials, and there was no significant difference in rainfall between these seasons, these factors could not be attributed to the variability of desiccated skin onset (in days). The significant temperature difference between the hot summer and cooler autumn season is one possible explanatory variable for the onset of this individual decomposition characteristics. Alternatively, racoon scavenging has previously been found to frequently occur in summer months and may also promote the onset of mummification [40].

Winter cadavers displayed the fewest decomposition characteristics but had the quickest average onset for 'blistering' and 'brown/black discoloration of the skin' compared to all other seasons. The colder temperatures and lower humidity of the winter trial may have caused the premature appearance of the "brown/black discoloration', which is considered another precursor for mummification [19] and marks the end of the 'early decomposition' phase in the TBS method [13]. However, it cannot be excluded that the covering conditions of winter cadavers could also have influenced these results. Given that most winter cadavers were covered, and spring cadavers were mainly uncovered, this could explain the quicker onset of these 'dry' decomposition traits, particularly if the non-absorbent plastic prevented rainfall from providing moisture for microbial activity 
[18]. Furthermore, winter cadavers had the longest duration of 'livor mortis' likely because cold temperatures delay the dissociation of oxygen from haemoglobin [4].

The variability in both the onset of individual decomposition characteristics and TBS scores between i) summer and autumn; ii) spring and iii) winter trials, has predominantly been explained by seasonal variation in humidity and to a lesser extent, temperature. The comparable decay rate between summer and autumn cadavers, despite significant temperature variance, attributes their exposure to similarly high humidity as the main accelerator of gross decomposition. However, temperature may be influential in determining the onset of some individual decomposition characteristics, particularly in relation to early precursors of mummification. Nonetheless, it is also recognised that decomposition variability in this study could also be explained by a combination of seasonal and non-seasonal variables such as scavenging access, entomology, and/or factors intrinsic to the human cadaver, which cannot be excluded in contributing to these results.

\section{$\underline{\text { TBS and TBSsurf Models }}$}

This paper has demonstrated that both Megyesi et al [13] and Moffatt et al [16] models were subject to confounding factors that limit their applicability to estimating the PMI in forensic casework. One such problem appears to be the estimation of ADDs developed retrospectively from the state of decay. This introduces a circularity into PMl estimation that has made existing models good at predicting estimated ADD but has opened two potential points of weakness: the first being the variance between estimated and actual ADD, and the second being the potential for low levels of dependence between temperature and PMI. While both models offer appealing promise in the prediction of estimated ADD, on repeated occasions this has confounded the expectations of later researchers seeking to test these models against actual measures of ADD [15], [18], [19], [23], [25]. Not only are estimated and actual ADD at variance in both models, but neither is capable of perceiving nor assessing the level of variance from actual ADD.

The results found that both TBS and TBSsurf models can predict estimated ADD with high confidence $\left(R^{2}=\right.$ 0.9). However, prediction of estimated ADD from TBS should be taken with caution, given that TBS should be the response (measurable) variable and not the explanatory variable [16]. This paper found no apparent relationship between actual $A D D$ values and the level of decay $\left(R^{2}=0.025\right)$ suggesting that temperature may not be as influential to the decay rate in the early post-mortem period, as previously thought. The actual ADD was significantly over-estimated at all TBS and TBSsurf scores derived across all seasons. This is not dissimilar to findings from other studies [18], [34], albeit some studies have found that the methods underestimate ADD [15], [23]. Therefore, when the estimated ADD from both methods is used to derive an estimated PMI there is a significant difference between the estimated PMI and the actual PMI, which currently confounds the use of ADD-derived PMI estimation models in forensic practice. This study found that TBSsurf had slightly improved predictability of the PMI over the TBS method between PMI days 5 to 7 only; albeit both models produced significant over-estimations of the PMI.

When exploring the effect of this error across different seasons, no seasonal trial could predict TBS or TBSsurf-estimated PMI with high confidence. Winter cadavers had the most significant over-estimations of PMI throughout the study duration. A likely explanation for this lies in disentangling the relationship between actual ADD and the known PMI. Errors were inherent when winter cadavers had been subject to $0^{\circ} \mathrm{C}$ or below 
freezing temperatures and accumulated degree days were not incurred. This was not surprising given that $0^{\circ} \mathrm{C}$ is considered to cease the decomposition processes [13]. This was reflected by the low and stagnant TBS and TBSsurf for January cadavers and the K-S results also indicated a significant deviation of winter TBS compared to all other seasons. It is therefore recommended that caution should be taken when using actual ADD values in PMI estimations when bodies are subject to $0^{\circ} \mathrm{C}$ or below freezing temperatures in forensic casework, particularly if it is not known how many days the body has been subject to $\leq 0^{\circ} \mathrm{C}$ temperatures.

Interestingly, spring cadavers had the slowest onset of the majority of decomposition variables and the lowest TBS and TBSsurf (excluding winter), but improved PMI estimations compared to all other seasons. This suggests that Megeysi et al [13] TBS method was most aligned with the decomposition rate of spring cadavers, which unveils two further discussion points. First, despite the similar temperature ranges of spring and autumn trials, autumn cadavers conferred the most extreme outliers of PMI prediction ( $>20-\leq 62$ days). This further supports the finding that temperature had a negligible effect on ADD-based PMI prediction models in this study. Second, scavenging (which is not accounted for in the TBS and TBSsurf methods) and scavenging-related advanced decomposition characteristics such as 'tissue sloughing' and bone exposure', had the slowest onset in spring cadavers. Conversely, early onset scavenging in summer and autumn cadavers resulted in higher, misrepresentative decomposition scores, which combined within significant errors between the actual ADD and the estimated ADD, caused significantly greater over-estimations of the PMl compared to spring cadavers.

While the TBS and TBSsurf methods could not be validated in the experimental conditions of this study, the independent recording of the onset of decomposition characteristics using visual reference guides $[35,36]$, served three complimentary purposes. First, it provided capability to assess the interrelationship between seasonal factors that may influence decomposition in a sophisticated manner that opens debate over variations in onset of decomposition characteristics per season at known PMls. Second, by observing the appearance of decomposition traits individually, it escaped the dominance of the co-associated decomposition variables that exist in the TBS method as stage-specific criteria; that has so far proved an ineffective method of decomposition recording [26] [31]. Third, the simple recording of the presence or absence of decomposition trait onset reduces the subjectivity of ambiguous terminology in the TBS method such as "some flesh still relatively flesh". The authors recommended individual recording of decomposition characteristics alongside any further experimental validation of the TBS/TBSsurf methods.

\section{Limitations of the Study}

The authors recognise that any predictive statistical model is by nature based upon an imperfect and imprecise set of retrospective data. While this study offers an opportunity to reinvestigate both the accuracy of ADD-based PMI estimation models in death investigation, and the relationship between temperature and the rate of human decomposition, it too is limited by the single geographical climate of Knoxville, Tennessee. In addition, this study investigated the decomposition pattern and rate over a relatively brief period of time (17 PMI days). While the different covering conditions did not appear to influence the trajectory of decomposition rate in this study, the temperature and humidity results are limited by the inability to exclude possible discrepancies between the 'micro-environments' created under the covered cadavers, and those 
uncovered cadavers exposed to the external environment. It is therefore recognised that observing past the early-post mortem period through to skeletonization may be required to confirm any continuity of the seasonal variation in decomposition patterns seen in this study. Furthermore, while all seasons were represented within the dataset and there were no significant differences in decomposition trajectory between months of the same season, not every month was included. February, March, November and December placements were missing from this study and need to be investigated with additional human cadavers to further confirm these results. While this study has focused on the effects of seasonality, there are a number of other environmental variables (such as wind speed, soil and exposure to sunlight or shade) and/or intrinsic factors (for example cause of death, body weight and clothing) that cannot be excluded in contributing to these results.

\section{Conclusion}

The onset and accumulation of decomposition characteristics of 26 donated human cadavers from the University of Tennessee varied significantly across the two seasons of summer and autumn, during the first 7 PMI days. This importantly encompasses the period in which entomology methods may not be applicable and early post-mortem biochemical markers (PBMs) are not yet routinely standardised for use in forensic practice. This study has found that humidity (which was comparable between summer and autumn) rather than temperature (which was significantly different) may be the pivotal driving force in the morphological appearance of decomposition in the early post-mortem period. However, temperature may still be influential in determining the specific onset of early mummification decay traits. This study was also the first to test the Moffat et al [16] revised TBSsurf system on human cadavers under experimental conditions finding, along with Megyesi et al [13] TBS method, inaccurate PMI estimations in the early post-mortem period. It is therefore suggested that ADD-based PMI estimation models are unlikely to have any potential value in forensic practice if the known ADD deviates significantly from the estimated ADD, which subsequently causes repeated validation failure under experimental conditions. While there is clear value in quantifying decomposition as a measure of trajectory and rate of this continuous process, the TBS and TBSsurf methods need to be significantly revised to account for seasonal expression and individual onset of decomposition characteristics to confer any potential forensic value. 


\section{References}

[1] H.T. Gelderman, L.Boer, T. Naujocks, A.C.M. IJzermans, W.L.J.M. Duijst, Estimation of the post-mortem interval estimation for human remains found on land in the Netherlands, Int. J. Legal Med. 132 (2019), pp. 863 873. https://doi.org/10.1016/i.forsciint.2013.03.037

[2] R.W. Mann, W.M. Bass, L. Meadows, Time since death and decomposition of the human body; variables and observations in case and experimental field studies. J. Forensic Sci. 35 (1990), pp. 103-111. https://doi.org/10.1520/JFS12806J

[3] Association of Chief Police Officers (ACPO), Murder Investigation Manual, 2006. Bramshill: National Centre for Police Excellence. Available online at: http://library.college.police.uk/docs/APPREF/murderinvestigation-manual-redacted.pdf

[4] C. Henßge, B.Madea, Estimation of the time since death in the early post-mortem period. Forensic Sci. Int. 144 (2004), pp. 167-175. https://doi.org/10.1016/i.forsciint.2004.04.051

[5] A.E. Donaldson, I.L. Lamont, Estimation of post-mortem interval using biochemical markers. Aust. J. Forensic Sci. 46 (2013), pp. 8-26. https://doi.org/10.1080/00450618.2013.784356

[6] J. Meurs, T. Krap, W. Duijst, Evaluation of postmortem biochemical markers: completeness of data and assessment of implication in the field. Sci. Justice. 59 (2019), pp. 177-180.

https://doi.org/10.1016/j.scijus.2018.09.002

[7] B. Madea, J. Ortmann, E. Doberentz, Estimation of the time since death-Even methods with a low precision may be helpful in forensic casework. Forensic Sci. Int. 302 (2019), 109879.

https://doi.org/10.1016/i.forsciint.2019.109879

[8] J. Amendt, C.S. Richards, C.P. Campobasso, R. Zehner, M.J.R. Hall, Forensic entomology: applications and limitations. Forensic Sci. Med. Path. 7 (2011), pp. 379-392. https://doi.org/10.1007/s12024-010-9209-2 [9] H.B. Reed, A study of dog carcass communities in Tennessee, with special reference to the insects. Am. Midl. Nat. 59 (1958), pp. 213-245. https://www.jstor.org/stable/2422385

[10] W. Rodriguez, W.M. Bass, Insect activity and its relationship to decay rates of human cadavers in East Tennessee. J. Forensic Sci. 28 (1983), pp. 423-432. https://doi.org/10.1520/JFS11524J

[11] A.A. Vass, W.M. Bass, J.D. Wolt, J.E. Foss, J.T Ammons, Time since death determinations of human cadavers using soil solution. J. Forensic Sci. 37 (1992), pp. 1236-1253.

https://www.jstor.org/stable/2422385

[12] A. Galloway, W.H. Birkby, A.M. Jones, T.E. Henry, B.O. Parks, Decay rates of human remains in an arid environment. J. Forensic Sci. 34 (1989), pp. 607-617. https://doi.org/10.1520/JFS12680J

[13] M.S. Megyesi, S.P. Nawrocki, N.H. Haskell, Using accumulated degree-days to estimate the postmortem interval from decomposed remains. J. Forensic Sci. 50 (2005), pp. 618-626.

https://doi.org/10.1520/JFS2004017 
[14] R.E. Adlam, T. Simmons, The effect of repeated physical disturbance on soft tissue decomposition are taphonomic studies an accurate reflection on decomposition? J. Forensic Sci. 52 (2007), pp. 10071014. https://doi.org/10.1111/j.1556-4029.2007.00510.x

[15] C.M. Fitzgerald, M. Oxenham, Modelling time-since-death in Australian temperate conditions. Aust. J. Forensic Sci. 41 (2009), pp. 27-41. https://doi.org/10.1080/00450610902935981

[16] C. Moffatt, T. Simmons, J. Lynch-Aird, An improved equation for TBS and ADD: establishing a reliable post-mortem interval framework for casework and experimental studies. J. Forensic Sci. 61 (2016), S201S207. http://dx.doi.org/10.1111/1556-4029.12931

[17] W.M. Bass, Outdoor decomposition rates in Tennessee, in: W.D. Haglund, M.H. Sorg (Eds.), Forensic taphonomy: the post-mortem fate of human remains. Boca Raton, CRC Press (1997), pp. 181-186.

[18] M.S. Archer, Rainfall and temperature effects on the decomposition rate of exposed neonatal remains. Sci. Justice. 48 (2004), pp. 35-41. https://doi.org/10.1016/S1355-0306(04)71683-4

[19] D.L. Cockle and L.S. Bell, The environmental variables that impact human decomposition in terrestrially exposed contexts within Canada. Sci. Justice. 57 (2016), pp. 107-117.

https://doi.org/10.1016/i.scijus.2016.11.001

[20] J. Meyer, B. Anderson, D.O. Carter, Seasonal variation of carcass decomposition and gravesoil chemistry in a cold (Dfa) climate. J. Forensic Sci. 58 (2013), pp. 1175-1182. https://doi.org/10.1111/1556$\underline{4029.12169}$

[21] M.N.S. Forbes, D.A. Finaughty, K.L. Miles, V.E. Gibbon, Inaccuracy of accumulated degree day models for estimating terrestrial post-mortem intervals in Cape Town, South Africa. Forensic Sci. Int. 296 (2019), pp. 67-73. https://doi.org/10.1016/i.forsciint.2019.01.008

[22] S.J. Marhoff, P. Fahey, S.L. Forbes, H. Green, Estimating post-mortem interval using accumulated degree-days ad a degree of decomposition index in Australia: a validation study. Aust. J. Forensic Sci. 1 (2016), pp. 24-36. https://doi.org/10.1080/00450618.2015.1021378

[23] C.L. Parks, A study of the human decomposition sequence in Central Texas. J. Forensic Sci. 56 (2011), pp. 19-22. https://doi.org/10.1111/j.1556-4029.2010.01544.x

[24] J. Myburgh, E.N. L'Abbé, M. Steyn, P.J. Becker, Estimating the post-mortem interval (PMI) using accumulated degree-days (ADD) in a temperate region of South Africa. Forensic Sci. Int. 229 (2013), pp. 165.e1 - 166.e6. https://doi.org/10.1016/..forsciint.2013.03.037

[25] J.K. Suckling, M.K. Spradley, K. Godde, A longitudinal study on human outdoor decomposition in Central Texas. J. Forensic Sci. 61 (2015), pp. 19-25. https://doi.org/10.1111/1556-4029.12892

[26] A.S. Ceciliason, M.G. Andersson, A. Lindström, H. Sandler, Quantifying human decomposition in an indoor setting and implications for postmortem interval estimation. Forensic Sci. Int. 283 (2018), pp. 180189. https://doi.org/10.1016/i.forsciint.2017.12.026 
[27] T.Simmons, Post-mortem interval estimation: an overview of techniques, in E.M.J. Schotsmans, N. Marquez-Grant, S.L. Forbes (Eds.), Taphonomy of human remains: forensic analysis of the dead and depositional environment. John Wiley \& Sons, Chichester, UK (2017), pp. 134-142.

[28] D.L. Cockle, L.S. Bell, Human decomposition and the reliability of a 'Universal' model for post mortem interval estimations. Forensic Sci. Int. 253 (2015), pp. 136.e1 - 136.e9.

https://doi.org/10.1016/i.forsciint.2015.05.018

[29] M. Connor, C. Baigent, E.S. Hansen, Testing the use of pigs as human proxies in decomposition studies. J. Forensic Sci. 63 (2017), pp. 1350-1355 https://doi.org/10.1111/1556-4029.13727

[30] A. Dautartas, M.W. Kenyhercz, G.M. Vidoli, L.M. Jantz, A. Mundorff, D.W. Steadman, Differential decomposition among pig, rabbit and human remains. J. Forensic Sci. 63 (2018), pp. 1673-1683.

https://doi.org/10.1111/1556-4029.13784

[31] G.R. Dabbs, M. Connor, J.A. Bytheway, Interobserver reliability of the total body score system for quantifying human decomposition. J. Forensic Sci. 61 (2016), pp. 445-451. https://doi.org/10.1111/1556$\underline{4029.12962}$

[32] M. Nawrocka, K. Frątczak, S. Matuszewski, S, Inter-rater reliability of total body score - A scale for quantification of corpse decomposition. J. Forensic Sci. 61 (2016), pp. 798-802.

https://doi.org/10.1111/1556-4029.13105

[33] G.R. Dabbs, J.A. Bytheway, M. Connor, Comparing the scoring of human decomposition from digital images to scoring using on-site observations. J. Forensic Sci. 62 (2017), pp. 1292-1296.

https://doi.org/10.1111/1556-4029.13409

[34] A. Ribéreau-Gavon, C. Rando, R.M. Morgan, D.O. Carter, The suitability of visual taphonomic methods for digital photographs: an experimental approach with pig carcasses in a tropical environment. Sci. Justice. 58 (2018), pp. 167-176. https://doi.org/10.1016/i.scijus.2017.12.001

[35] J. Dix, M. Graham, Time of death, decomposition and identification: an atlas. Boca Raton, CRC Press, (2000)

[36] J. Dix, Colour atlas of forensic pathology. Boca Raton, CRC Press, (2000), pp. 2-18.

[37] J. Frey, An exact Kolmogorov-Smirnov test for whether two finite populations are the same. Statist. Probab. Lett. 116 (2016), pp. 65-71. https://doi.org/10.1016/i.spl.2016.04.016

[38] M.S. Micozzi, Experimental study of postmortem change under field conditions: effects of freezing, thawing and mechanical injury. J. Forensic Sci. 31 (1986), pp. 953-961. https://doi.org/10.1520/JFS11103J. [39] A.M. Daurtartas, The effect of various coverings on the rate of human decomposition. Masters Thesis, University of Tennessee, 2009. Available online at: https://trace.tennessee.edu/utk gradthes/69 [40] J. Yangseung, L. M. Jantz, J. Smith, Investigation into seasonal scavenging patterns of racoons on human decomposition. J. Forensic Sci. 61(2016), pp. 467-471

[41] D.A. Finaughty, A.G. Morris, Precocious natural mummification in a temperate climate (Western Cape, South Africa). Forensic Sci. Int. 303 (2019), 109948. https://doi.org/10.1016/i.forsciint.2019.109948 
